\title{
Fragments Extraction and Segmentation Based on Three Frames Difference and Modified Otsu
}

\author{
Zeqing Liu ${ }^{1, a}$, Yurong Zhang ${ }^{1, b}$, and Jianxin Zhao ${ }^{1, c}$ \\ ${ }^{1}$ Department of Artillery Engineering, Ordnance Engineering College, Shijiazhuang 050003, China \\ aliuzeqing320123@sina.com, b zhangoec1@163.com, 'zjx08@mail.tsinghua.edu.cn
}

Keywords: Otsu, image extraction, three frames difference, threshold segmentation

\begin{abstract}
This paper present a method for the extraction and segmentation of small targets in image sequence captured in static explosion test, whose background is complex with numerous interference. Three frames difference method is adopted to process the original image sequence to obtain the foreground image. Since the foreground image is heavily contaminated by noise, so the gray square enhancement method is used to increase the contrast between the noise and the target. The modified Otsu method is employed to obtain the global optimum threshold, and the segmentation of the foreground image is accomplished. Finally, morphological processing is performed, and the noise of the isolated points is eliminated, and the weakening of the target in the process of differential and segmentation is resumed. Through a series of processing, the extraction of fragments in static explosion test image sequence is completed, and the trajectories of the fragments are obtained.
\end{abstract}

\section{Introduction}

High speed photography is an important method to measure fragment velocity in range static explosion test [1]. Using multiple high-speed cameras to shoot the movement of fragments from different angles, we can get the image sequences of fragments. The trajectories of fragments can be restored by the extraction of image sequences, and the velocity of fragments can be calculated with the trajectories known. The accuracy of the velocity obtained from the trajectories is determined by accuracy of the trajectories extracted from the image sequences. Therefore, it's important to study methods to extract the trajectories with high-precision.

As we all know, in the field static explosion test, the background is complex and there are numerous interference factors. What's more, the fragment's small in shape but large in number. All these factors make it difficult to extract the trajectories of fragments accurately. At present, the main methods of moving object extraction include optical flow method, the adjacent frame difference method, and the background subtraction method [2]. The optical flow method is based on the pixels' vector characteristics of image sequences for motion region detection, and it's accuracy for detection is affected by target characteristics, light illumination, target's velocity and noise and it's not suitable for the extraction of fragments from static explosion image sequences[3]. Neighbor frame difference method can extract the foreground of moving object by the gray difference of adjacent frame on image sequences which is insensitive to illumination change and noise interference [4], so it can be used to the extraction of the fragments. Three frames difference method is a modification of neighbor frame difference method, which can not only achieve the purpose of target extraction but also target enhancement.

\section{Foreground extraction method}

Neighbor frame difference method. In the shooting process, if the camera's position and attitude remain unchanged, it's considered that the background of the image sequence captured is constant and only the gray values of the moving target are changed. The difference between two adjacent frames, which represents the gray matrix of moving targets' foreground, can be found by gray matrices' subtraction. This is the basic idea of the neighbor frame difference method [5]. 
Three frames difference based on square enhancement. Because of the changes of illumination and camera current and other factors, there is a certain noise in every image in the sequence. After neighbor frame difference operation, the small target is easy to be drowned in the moving targets' foreground. That is to say, the effect is not ideal by using the neighbor frame difference method. Therefore, the three frame difference method based on the square enhancement is proposed to improve the foreground extraction effect and increase the contrast of target and noise.

Let $f_{k}(i, j) 、 f_{k+1}(i, j) 、 f_{k+2}(i, j)$ be three adjacent images in the image sequence. The differences between each of the two images of the three adjacent images can be described as followed:

$$
\begin{aligned}
& D_{k 1}(i, j)=\left|f_{k}(i, j)-f_{k+1}(i, j)\right| \\
& D_{k 2}(i, j)=\left|f_{k}(i, j)-f_{k+2}(i, j)\right| \\
& D_{k 3}(i, j)=\left|f_{k+1}(i, j)-f_{k+2}(i, j)\right|
\end{aligned}
$$

$D_{k 1}(i, j), D_{k 2}(i, j), D_{k 3}(i, j)$ are the foreground gray matrices of moving targets.

Adding the three foreground gray matrices together, we can get the adding foreground gray matrix

$$
D_{k}(i, j)=D_{k 1}(i, j)+D_{k 2}(i, j)+D_{k 3}(i, j)
$$

Because the noise is random, the noise of different points at the same time and the same point's noise at different time are all different. But the positions of the targets in three images are determined. So, by adding the three foreground gray matrices together, the gray of targets' position can be enlarged. That's to say, That is to say, the contrast of the targets' gray value and the noise has been enhanced. However, this kind of superposition enhancement is a linear operation, and the contrast is not obvious enough for targets' extraction.

Square processing is a nonlinear enhancement method, which can increase the contrast between the parameters in quadratic form. Therefore, the method of square processing is adopted to square every element of the foreground gray matrix. After the square processing, we can get the enhanced foreground gray image $G_{k}$

$$
G_{k}(i, j)=G_{k}^{2}(i, j)
$$
Fig.1.

The three frames difference based on square enhancement processing can be described as the

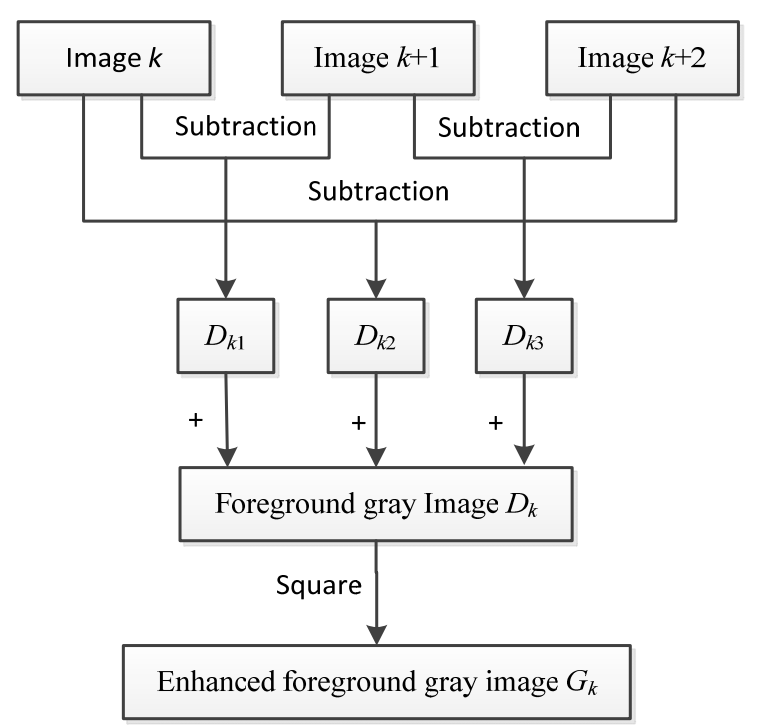

Fig. 1 Three frames difference based on square enhancement

\section{Binarization threshold determining by modified Otsu}

Otsu. After the above two steps, the contrast between the target foreground and the noise is greatly enhanced. In order to segment the target foreground from the noise, what's needed to do is set a threshold. The most commonly used method to determine the threshold is Otsu. The algorithm selects 
the threshold to maximize the variance of the class between the target and the background. The bigger the variance is, the more different two parts of the image are [6].

Setting the number of pixels of the image is $N$, and the gray range is $[0, L-1]$. The number of the gray level $i$ which belongs to $[0, L-1]$ is $n_{i}$, and its probability is

$$
\begin{aligned}
& p_{i}=n_{i} / N \\
& \sum_{i=0}^{L-1} p_{i}=1, \quad \theta=\sum_{i=0}^{t} p_{i},
\end{aligned}
$$

The gray value of the image is divided into two categories, e.g. class $C_{1}$ and class $C_{2}$. The gray value of class $C_{1}$ belongs to $[0, t]$, and the gray value of class $C_{2}$ belongs to $[t, L-1]$. Then the average of the gray value of image and class $C_{1}$ and class $C_{2}$ can be described as followed:

$$
\mu=\sum_{i=0}^{L-1} i p_{i}, \quad \mu_{1}=\sum_{i=0}^{t} i p_{i} / \theta, \quad \mu_{2}=\sum_{i=t}^{L-1} i p_{i} /(1-\theta)
$$

From the formula (7), we can know that :

$$
\mu=\theta \mu_{1}+(1-\theta) \mu_{2}
$$

Variance between two classes can be defined as

$$
\sigma^{2}=\theta\left(\mu-\mu_{1}\right)^{2}+(1-\theta)\left(\mu_{2}-\mu\right)^{2}=\theta(1-\theta)\left(\mu_{1}-\mu_{2}\right)^{2}
$$

The optimal threshold is defined as $t$, which can make the variance $\sigma^{2}$ maximum.

But, if there are uneven illuminations, burst noises or great changes in background, the threshold calculated by Otsu is not the optimal. And the region the threshold segments not only includes the target area, but also the background with great changes [7]. In order to eliminate the misjudgment region caused by gray changes, it's needed to modify the Otsu.

Modified Otsu. After the extraction and enhancement processing, the gray value of the target has been improved greatly. It's found that the mean value of the targets area's gray is significantly higher than that of the noise and the noise and the target areas and noises are divided into several small regions. That's to say, the foreground image can be segmented into several small regions. By studying the regions separately, the thresholds of every region can be found out. Since the target area's gray value is higher than the noises, the global optimal threshold can be obtained by solving the maximum value of the threshold of all regions. The specific process flow is shown in Fig 2.

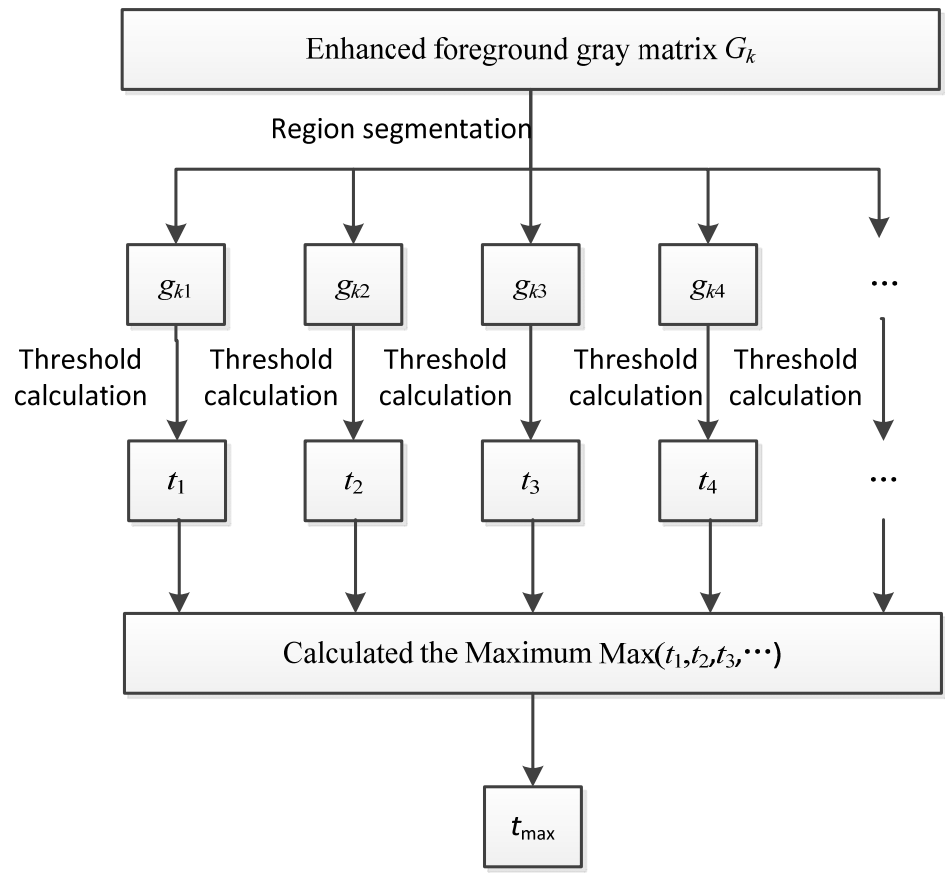

Fig. 2 Computation of global optimum threshold 


\section{Morphological processing}

In the process of difference and segmentation, the pixels of the target are lost, and there are some holes and isolated points. So the foreground image is further processed to eliminate holes and isolated points.

Isolated point elimination. It's known that the target area is the aggregation of multiple pixels in a certain range, but the noise is the singular point. So we can set a search range, target gray value and target number, and search the number of pixels, whose gray value is larger than target gray value, around each pixel. If the number is less than the target number, the pixel will be identified as noise.

Expansion processing. The expansion processing makes the boundary expand outward. It can merge the points around the boundary into the target area, and combine the neighboring regions. With the expansion processing, the holes caused by expansion and segmentation can be repaired, and the contour of the target areas can be recovered to a certain extent.

\section{Results}

Taking an image sequence static of explosion test as example to process. There are two fragments in the images as shown in Fig 3. The image sequence is processed by neighbor frame difference method and three frames difference method based on square enhancement separately, and segmented by Otsu. The results are shown in Fig 4 (a) and (b). After that, the sequence processed by three frames difference method based on square enhancement is respectively segmented by Otsu and modified Otsu. The results are shows as Fig 5 (a), (b).

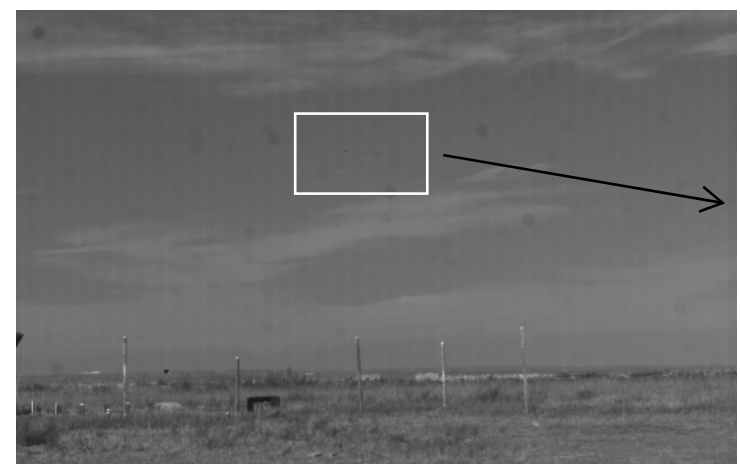

(a)

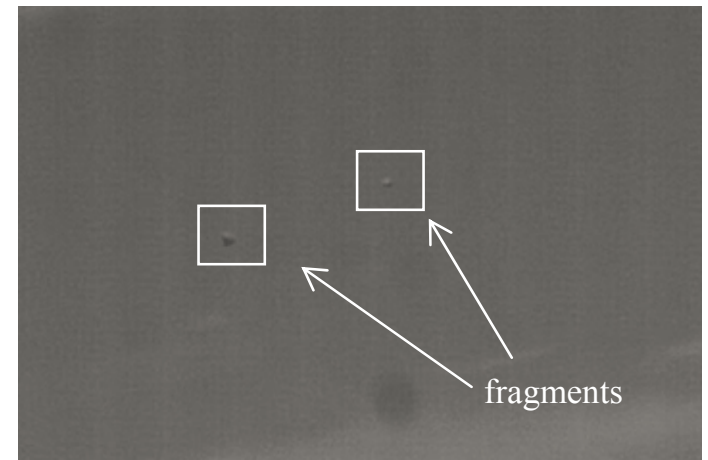

(b)

Fig.3 Fragments in stasic explosion image

(a)Static explosion image

(b)Fragments

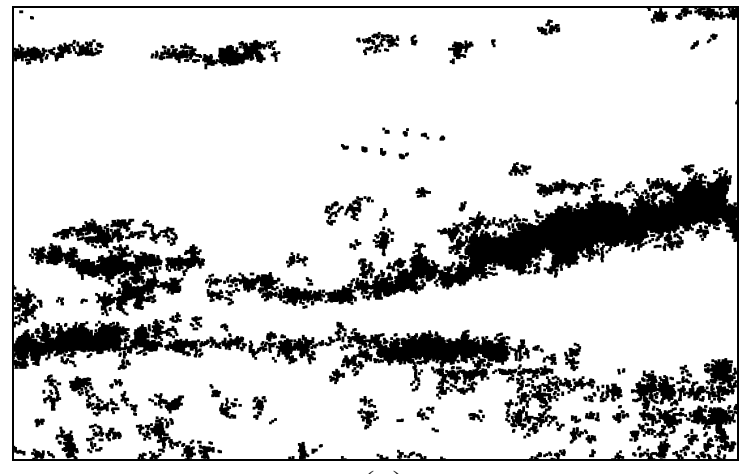

(a)

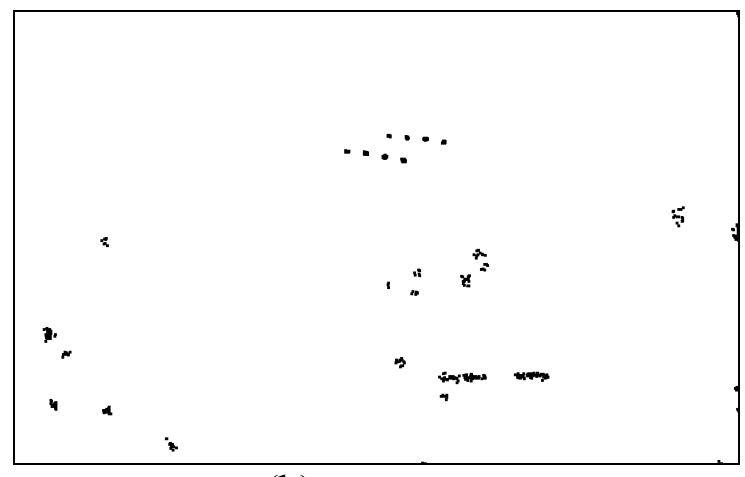

(b)

Fig.4 Image extraction results

(a) Result of neighbor frame difference

(b)Result of three frames difference method based on square enhancement 


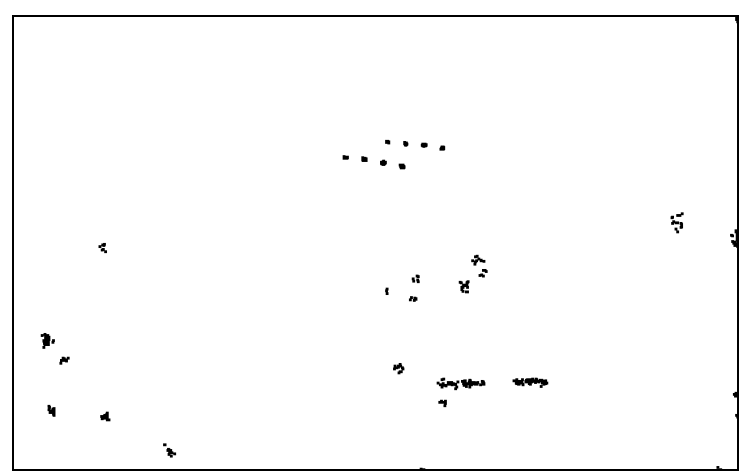

(a)

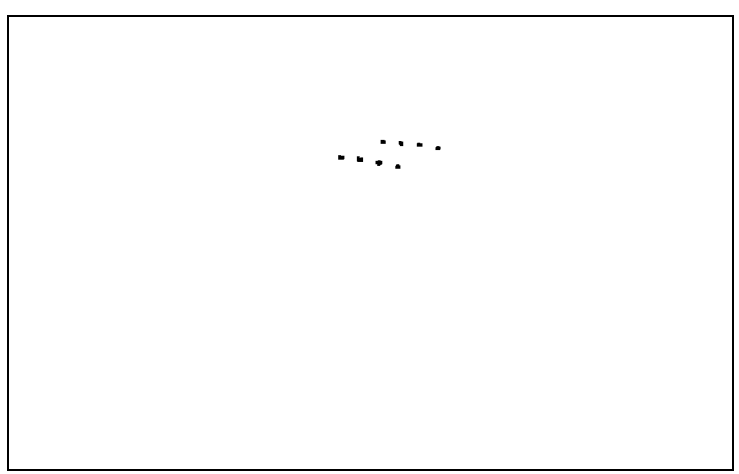

(b)

Fig.5 Image segmentation results

(a) Result of Otsu

(b)Result of modified Otsu

From Fig.4 (a), (b) we can find that, the image extracted by frame difference method has many noises, while the one extracted by three frames difference method based on square enhancement has less noises. It is obviously that, the three frames difference method based on square enhancement can greatly increase the contrast between target and noise. Comparing to Fig.5 (a), we can figure out that Fig.5 (b) has a better segmentation result. The noises in several other regions were elimated, overcoming the problem of misjudgment.

\section{Summary}

In this paper, the method for the extraction and segmentation of small targets in static explosion test image sequence has been introduce. The process consists of three phases, e.g. extraction , segmentation and morphological processing.

With the method of three frames difference based on square enhancement, the contrast between the targets'value and noise is enhanced greatly. And the modified Otsu can segment the targets from the noise better, solving the problem of misjudgment.

From the data processing results, it can be seen that the method can exclude the interference of background, and extract position of fragments accurately. The extraction and segmentation results can meet the requirements of the fragments position calculation in the image.

\section{References}

[1] Huaning Liu, Yu Zheng, Wenbin Zheng, etc., Velocity measurement method of projectiles based on high speed photography technology, Ordnance Industry Automation, 33(11), (2014), 71-74.

[2] Wang L, Hu W, Tan T, Recent developments in human motion analysis, Pattern Recognition, , 36(3), (2003), 585-601.

[3] Jianfei Liu, Kalpathi R. Subramanian, Terry S. Yoo, An optical flow approach to tracking colonoscopy video, Computerized Medical Imaging and Graphics, 37(3), (2013), 207-223.

[4] Kim C, Hwang J N, Fast and automatic video object segmentation and tracking for content-based application, IEEE Trans on Circuits and Systems,1(2),(2002),122-129.

[5] Jain R, Nagel H, On the analysis of accumulative difference of picture from image sequences of real world scenes, IEEE Trans. PAMI, (1979), 206-214.

[6] N Otsu, A threshold selection method from gray level histograms, IEEE Trans on SMC, 9(1), (1979), 62-69.

[7] Jin Lisheng, Tianlei, An improved Otsu image segmentation algorithm for path Mark detection under variable illumination, Proceedings IEEE of intelligent Vehicles Symposium, (2005),840-844. 